\title{
ANALOgY FAUlT MODEL For BIQUAD FILTER BY USING VECTORISATION METHOD
}

\author{
Gurunadha.Ravva $^{1}$ and k.Babulu ${ }^{2}$ \\ ${ }^{1}$ Department of ECE, JNTUK-UCEV, Vizianagaram, Andhra pradesh, India \\ ${ }^{2}$ Department of ECE, JNTUK kakinada Andhra Pradesh, India
}

\begin{abstract}
In this paper a simple shortcoming model for a CMOS exchanged capacitor low pass channel is tried. The exchanged capacitor (SC) low pass channel circuit is modularized into practical macros. These useful macros incorporate OPAMP, switches and capacitors. The circuit is distinguished as flawed if the recurrence reaction of the exchange capacity does not meet the configuration detail. The sign stream chart (SFG) models of the considerable number of macros are investigated to get the broken exchange capacity of the circuit under test (CUT). A CMOS exchanged capacitor low pass channel for sign recipient applications is picked as a case to exhibit the testing of the simple shortcoming model. To find out error is to calculate EIGEN values and EIGEN vectors is to detect the error of each component and parameters
\end{abstract}

\section{INTRODUCTION}

Design of an analog circuit fault models is a challenging research problem, because of the long fault simulation time and unverified fault assumption. The major practical issues in developing an analog fault model include Zero noise margin, Nondeterministic transfer function, Too long fault simulation time and complex causal variations. Analog faults are of two types soft faults and hard faults. Parametric faults are due to change in the components of the parameter. Like resistor values and capacitance values .

A hard fault occurs due to short or opens wire. Most of the CMOS analog circuits are implemented by using switched capacitor (SC) circuits. SC circuits have the advantages of responses, good linearity wide dynamic ranges and accurate frequency .

Recently a static linear behaviour (SLB) analog fault model is proposed for linear and time invariant sampled SC circuits [1-2].Here consider a fixed z-domain transfer function. The SLB fault model covers not only parametric faults such as open loop gains of the OPAMP and capacitor ratios of the capacitors but also the catastrophic faults of the OPAMP, switches and capacitors of the CUT. This fault model deals with multiple faults when compare to the conventional fault model.

To minimizing the number of switches in a circuit the switch sharing technique is used and to improve the filter characteristics two methods are used dynamic range scaling and minimum capacitor scaling.

In this paper is organized as follows. Section 2 discusses the SFG's that are helpful in SLB fault model. Section 3 depicts the analysis that was made on the CUT, fault injections and their results 
are shown. Section 4. Depicts the switched capacitor common mode feedback circuit description and finally conclusion from obtained results in section 5 .

\section{STATIC LINEAR BEHAVIOUR FAULT MODEL}

The important parameters that effect the faultiness of the CUT are, OP-AMPS: For a given OPAMP, the open loop gain should have minimum specifications and the offsets have the largest specification.

Capacitors: Ratio of the capacitors to the corresponding feedback circuit capacitance in the CUT. Switches: Delayed switched capacitor branch and the delay free switched capacitor branch contain the switches has an additional fault.

The schematic circuit diagram for switched capacitor biquad filter is drawn as shown in Fig.1

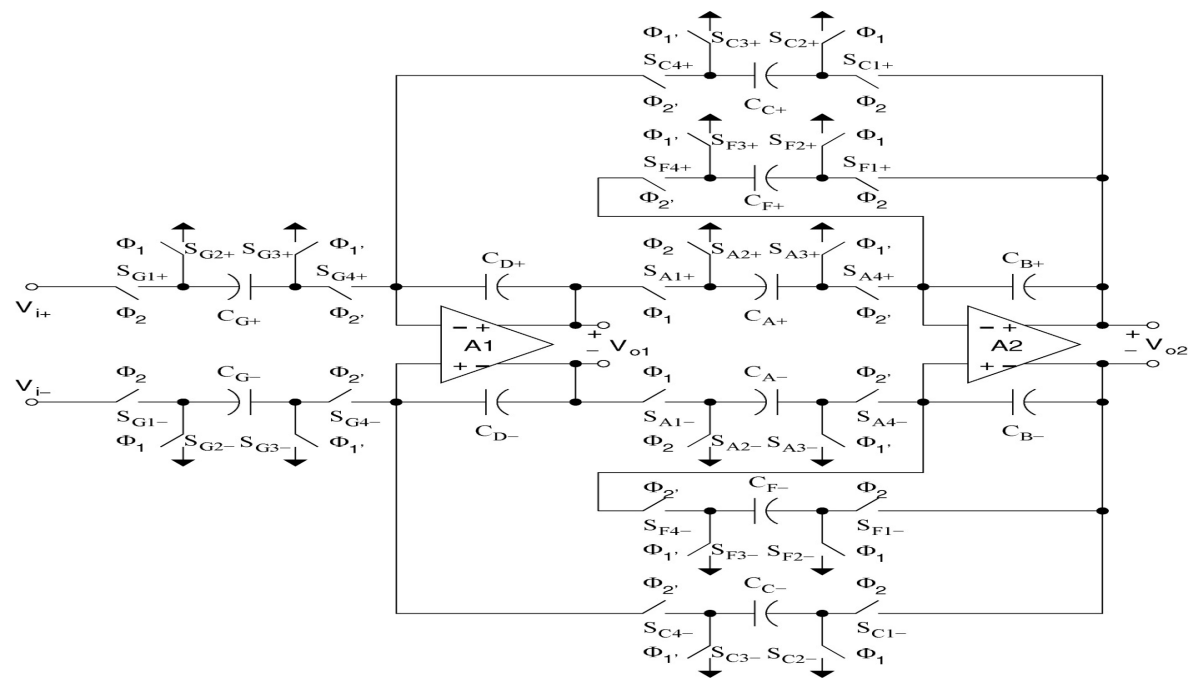

Fig.1 Schematic circuit of SC biquad filter

The specifications of the circuit are clock frequency of $6.144 \mathrm{MHz}$ andband pass from 0 to $20 \mathrm{KHz}$, , stop band attenuation is higher than $40 \mathrm{~dB}$ and pass band ripple is $<1 \mathrm{~dB}$ the remaining specifications are summarized in Table .1

\begin{tabular}{|c|c|}
\hline Parameter & Specification values \\
\hline$C_{A}$ & $1.706 \mathrm{pF}$ \\
\hline$C_{B}$ & $3.293 \mathrm{pF}$ \\
\hline$C_{C}$ & $1.706 \mathrm{pF}$ \\
\hline$C_{D}$ & $1 \mathrm{pF}$ \\
\hline$C_{F}$ & $3.259 \mathrm{pF}$ \\
\hline$C_{G}$ & $1.011 \mathrm{pF}$ \\
\hline $\mathrm{A} 1$ & $80 \mathrm{~dB}$ \\
\hline $\mathrm{A} 2$ & $80 \mathrm{~dB}$ \\
\hline
\end{tabular}

Table 1. Parameters of the switched capacitor circuit 
The signal flow graph for the fully differential SC biquad filter of CUT is shown in Fig.2

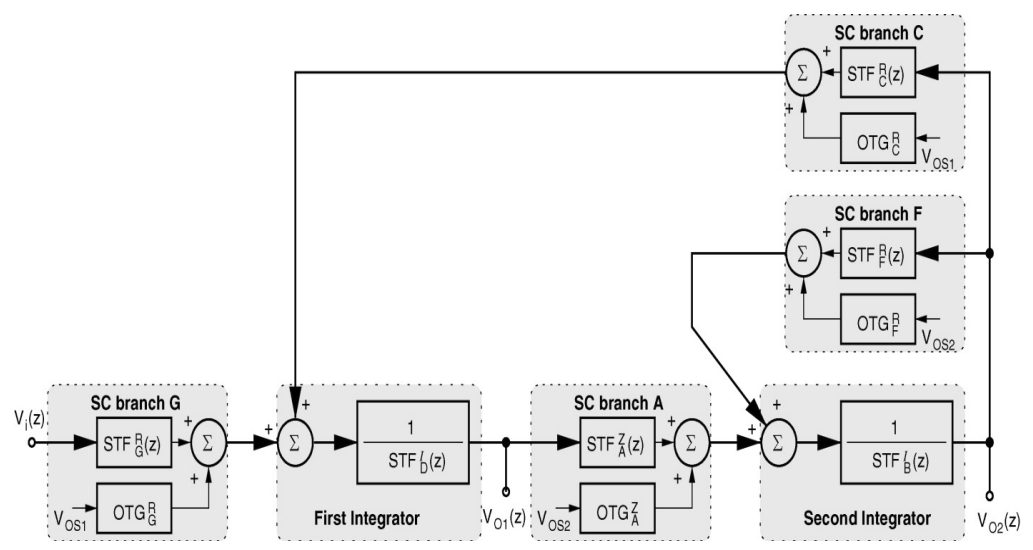

Fig.2 Signal flow graph of the CUT

By using this signal flow graph analysis calculate the overall input and output relationships of the circuit under test.

$$
V_{02}(z)=S T F_{H_{2}}(z) V_{i}(z)+O T G_{12} V_{O \alpha_{1}}+O T G_{22} V_{O s_{2}} \quad \cdots E q(1)
$$

Eq. (1) contains the signal transfer function (STF) and basic blocks of the SC biquad filter while the OTG's refer to the offset transfer gains of the corresponding stages the biquad. By considering Eq. (1) and SFG models, the design parameters of the STF of the biquad filter is

$$
V_{02}(z)=\left(-\left(C_{A} / C_{B}\right),\left(C_{G} / C_{D}\right), Z^{-1}, V_{i}(z)\right) / D E N(z) \quad \cdots E q(z)
$$

Where the denominator term is expressed as:

$$
D E N(z)=\left(1+\left(C_{F} / C_{B}\right)\right)-\left(2-\left(C_{A} C_{C} / C_{B} C_{D}\right)+C_{F} / C_{B}\right) Z^{-1}+Z^{-2} \quad \cdots E q(3)
$$

Assuming that the fault free Op-Amps have zero offsets. There are different kind of faults can be injected in the biquad. As we seen from the Eq. (2) and Eq. (3) the faults in the CUT changes the values of the coefficients of the signal transfer function which alter nothing but the capacitor ratios. Hence it is important to maintain the capacitor ratio instead of the absolute capacitance values. Capacitors may introduce a parametric fault.

soft faults in Op-Amps include its OPG and input referred offset while the hard faults in OpAmps are fatal due to its sensitivity in the design.. Faults due to switches are mostly same as in digital circuits i.e., stuck-at faults.

The fault free response of the CUT is simulated using MENTOR GRAPHICS tool with $0.35 \mu \mathrm{m}$ technology. The result is as shown in the Fig. 4. Here different simulated waveforms for different values of Op-Amp open loop gains. In this simulation obtain the outputs at both the output nodes in the two stage $\mathrm{SC}$ biquad filter. 
International Journal on Cybernetics \& Informatics (IJCI) Vol. 5, No. 4, August 2016

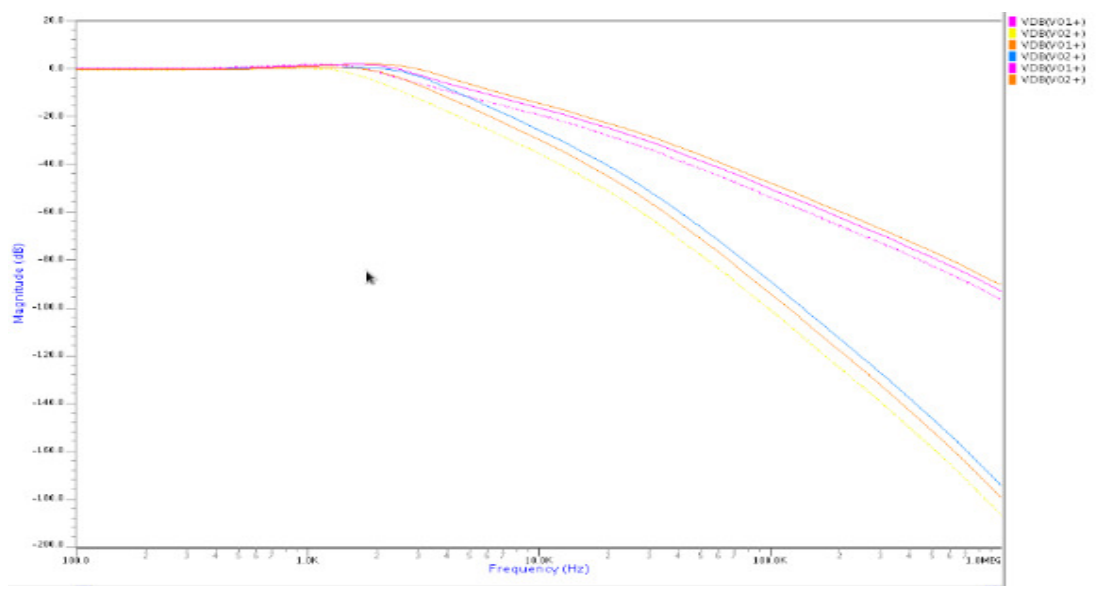

Fig. 3. Response of the Fault free CUT

As seen from Fig.3 outputs $V_{02}$ and $V_{01}$ illustrating outputs at first and second stages. Using EZ Wave tool in MENTOR GRAPHICS, compare the responses of the CUT for different Op-Amp open loop gains. This response can be used to verify the faultiness our CUT just by comparing the results of the faulty and fault free CUT's.

\section{FAult InJeCtion To The Cut}

Fig.4 depicts the schematic of the fault inject able Fleischer-Laker SC biquad. Like most of the analog circuit designs for IC, the CUT design is fully differential for better noise immunity and common mode interference rejection. The simulation was done on $0.35 \mu \mathrm{m}$ CMOS technology available in Mentor Graphics. Conventional folded cascode topology is used for implementing the Op-Amps. The design values of the capacitors in the fault injected CUT are given in Table 2 


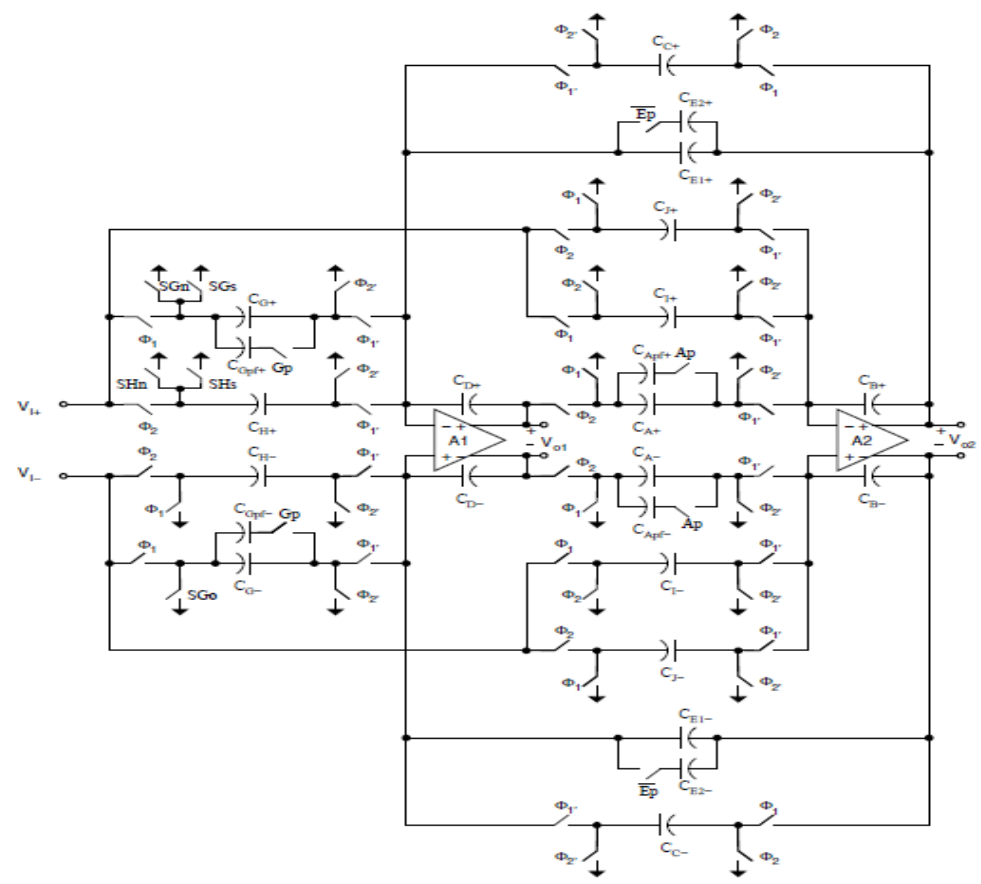

Fig. 4. Fault injected Schematic of SC biquad

SC biquad is used to realize a low-pass Butterworth filter whose band pass is $20 \mathrm{kHz}$ with frequency of sampling $2.5 \mathrm{MHz}$ and with stop band frequency of $400 \mathrm{kHz}$ and stop band attenuation is $30 \mathrm{~dB}$.These are the design specification of this filter.

The SLB analog fault model covers both parametric and catastrophic faults. Parametric faults are the results of parameter deviations of components due to process, voltage, and temperature variations. Capacitance values deviations are typical parametric faults in SC circuits.

Hence, add two differential capacitor pairs $C_{A p f}$ and $C_{E p f}$ to the biquad and decompose $C_{E}$ into two capacitors $C_{E 1}$ and $C_{E \text { I. }}$. By issuing the control signals Ap and $\mathrm{Gp}$.. Similarly, activating the control signal Ep will reduce the effective capacitance of $C_{E}$ by $C_{E 2}$ to model another kind of parametric fault. The capacitance values of these capacitors are listed in Table 2.

\begin{tabular}{|c|l|l|}
\hline Parameters & Designated ratio & Absolute value $(\mathrm{pF})$ \\
\hline$C_{A}$ & $0.20289855 C_{B}$ & 1.288 \\
\hline$C_{B}$ & $1 C_{\Xi}$ & 6.348 \\
\hline$C_{C}$ & $0.07246376 C_{D}$ & 0.460 \\
\hline$C_{D}$ & $1 C_{D}$ & 6.348 \\
\hline$C_{E}=C_{E 1}+C_{E 2}$ & $0.81159420 C_{D}$ & 5.152 \\
\hline$C_{G}$ & $0.10144927 C_{D}$ & 0.644 \\
\hline$C_{H}$ & $0.0289855 C_{D}$ & 0.184 \\
\hline$C_{I}$ & $0.00362318 C_{B}$ & 0.023 \\
\hline$C_{J}$ & $0.00724637 C_{B}$ & 0.046 \\
\hline
\end{tabular}


International Journal on Cybernetics \& Informatics (IJCI) Vol. 5, No. 4, August 2016

\begin{tabular}{|c|l|l|}
\hline$C_{A \psi f}$ & $0.4057971 C_{E}$ & 2.576 \\
\hline$C_{E 1}$ & $0.4057971 C_{D}$ & 2.576 \\
\hline$C_{E 2}$ & $0.4057971 C_{D}$ & 2.576 \\
\hline$C_{G p f}$ & $0.20289854 C_{D}$ & 1.288 \\
\hline
\end{tabular}

Table 2. Capacitor Ratios and Values of the CUT

To verify the deductions of the SLB fault model about the catastrophic faults, add three switches including SGs, SHs, and SGo to the design. The aspect ratios of the switches SGs and SHs are designed to be much larger than those of the switches SGn and SHn which are in parallel with them.

By keeping SGs or SHs turn-on, a short fault is injected to the corresponding switch. The design allows injecting a stuck-open fault to the biquad, too. The switch SGo can be turn off for injecting the stuck-open fault.

\section{Fifth ORder Switched CAPacitor Low PASS FiLter}

The schematic circuit diagram of fifth order SC low pass filter is shown in Fig.

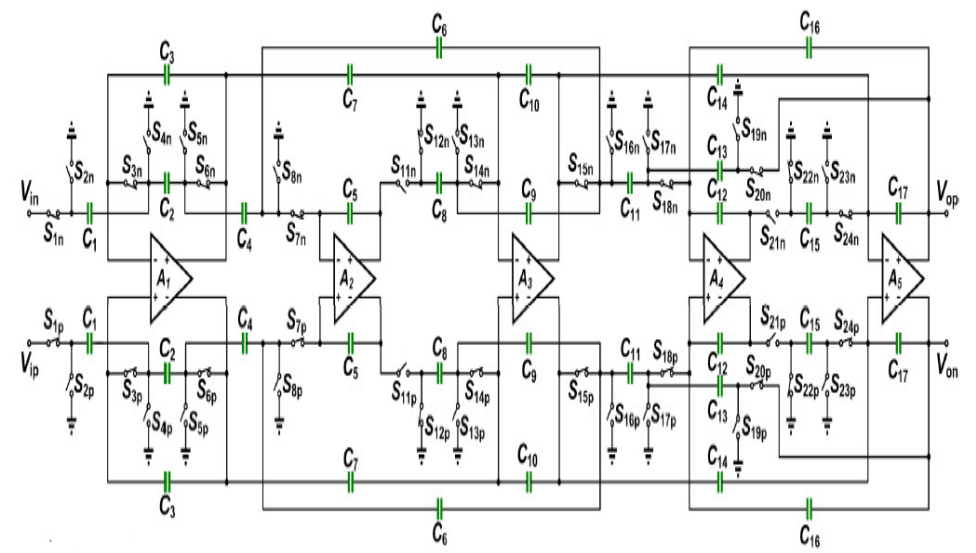

Fig.5 Schematic circuit of fifth order low pass filter

There are two approaches to design the SC low pass filter is cascade and ladder filter design. The cascade approach is done here. To design the higher order low pass filter, it consists of first and two second order circuits and the transfer function can be obtained from the second order continuous time low pass filter by using SFG analysis. The capacitance value of the capacitors of the circuit is listed in Table 3 . 
International Journal on Cybernetics \& Informatics (IJCI) Vol. 5, No. 4, August 2016

Table 3. Capacitor Values of the fifth order SC low pass filter

\begin{tabular}{|c|c|c|}
\hline Capacitor $(\mathrm{pF})$ & Dynamic range scaling & Minimum capacitor scaling \\
\hline$C_{1}$ & 1.23 & 0.41 \\
\hline$C_{2}$ & 1.2 & 0.4 \\
\hline$C_{3}$ & 3.42 & 1.14 \\
\hline$C_{4}$ & 2.25 & 0.75 \\
\hline $\mathrm{C}_{5}$ & 3.81 & 1.27 \\
\hline$C_{6}$ & 2.25 & 0.75 \\
\hline$C_{7}$ & 1.2 & 0.4 \\
\hline$C_{\mathrm{g}}$ & 2.25 & 0.75 \\
\hline$C_{\mathrm{g}}$ & 1.89 & 0.63 \\
\hline$C_{10}$ & 3.81 & 1.27 \\
\hline$C_{11}$ & 5.07 & 1.69 \\
\hline$C_{12}$ & 7.5 & 2.5 \\
\hline$C_{13}$ & 5.13 & 1.71 \\
\hline$C_{14}$ & 1.62 & 0.54 \\
\hline$C_{15}$ & 5.13 & 1.71 \\
\hline$C_{16}$ & 1.2 & 0.4 \\
\hline$C_{17}$ & 7.5 & 2.5 \\
\hline$C_{\max / \min }$ & & 6.25 \\
\hline
\end{tabular}

\section{VECTORISATION METHOD:}

The minimum and maximum principle that the matrix Eigen values varies with the change of matrix elements. In this way the Eigen values of matrix $A$ and the elements of the parametric fault set of CUT can be put one-to-one correspondence. So the proposed method is available to implement the faults diagnosis for analog circuits. The algebraic theory guarantees the correctness of this approach. The fault diagnosis procedure of the proposed method is described as follows.

Numbered the $n$ components required for fault diagnosis from 1 to $n$.

$$
\left[\begin{array}{cccc}
A_{11} & A_{12} & \ldots & A_{1 n} \\
A_{21} & A_{22} & \ldots & A_{2 n} \\
\vdots & \vdots & \ddots & \vdots \\
A_{n 1} & A_{n 2} & \ldots & A_{n n}
\end{array}\right]\left[\begin{array}{c}
v_{1} \\
v_{2} \\
\vdots \\
v_{n}
\end{array}\right]=\left[\begin{array}{c}
w_{1} \\
w_{2} \\
\vdots \\
w_{n}
\end{array}\right]
$$

Where as each row

$$
w_{i}=A_{i 1} v_{1}+A_{i 2} v_{2}+\cdots+A_{i n} v_{n}=\sum_{j=1}^{n} A_{i j} v_{j}
$$


If it occurs that $v$ and $w$ are scalar multiples, that is if

$$
A v=\lambda v
$$

then $v$ is an eigenvector of the linear transformation $A$ and the scale factor $\lambda$ is the eigenvalue corresponding to that eigenvector. Equation (1) is the eigenvalue equation for the matrix $A$.

Equation (1) can be stated equivalently as

$$
(A-\lambda I) v=0,
$$

Step-1:The output response signal of the actual is CUT stimulated by the same signal used in the simulation of software circuit is measured.

Step-2: According to the sample results, the output response matrix is generated, and the maximal and minimal eigenvalues $\left(\boldsymbol{v}_{\max }\right.$ and $\left.\boldsymbol{v}_{\min }\right)$ are calculated out.

Step-3: Assuming that $\boldsymbol{v}_{\max }$ is produced by each fault cases, respectively, the potential parameter value $x_{i}$ of the $i$ component is calculated out from

\section{$X_{i}=\left(V_{m x i}-b_{m x i}\right) / k_{m x i}, 1 \leq i \leq n .-\cdots-\cdot-\cdot(1 a)$}

The potential minimal eigenvalue $\boldsymbol{v}_{\boldsymbol{m} n \boldsymbol{i}}$ can be calculated out from the linear equation of Eigen matrix stated above and the above potential parameter $\mathbf{v}_{\mathrm{mni}}=\mathbf{k}_{\mathrm{mni}} \times\left(\mathbf{v}_{\mathrm{max}}-\mathbf{b}_{\mathrm{mxi}}\right) \mathbf{k}_{\mathrm{mxi}}+\mathbf{b}_{\mathrm{mni}}, \mathbf{1} \leq \mathbf{i} \leq$ n.-------(1b)

Step-4: Now the error values are calculated out as $E=[e 1, e 2, \ldots, e n]$. The element $e i$ of $E$ is calculated out as follows:

$$
\mathbf{e}_{\mathbf{i}}=\left|\mathbf{V}_{\mathbf{m n i}}-\mathbf{V}_{\mathbf{m i n}}\right| \text {. }
$$

Fault diagnosis. Only one of the $n$ assumptions in step 3)is correct, so only one of the $n$ results obtained from (1b) in step 3) is closest the minimal Eigen value $\boldsymbol{v}_{\min }$ obtained in step 1, i.e., the smallest element of $E$ corresponds to the correct assumption. If the subscript of the smallest element of $E$ is $m$, the CUT has fault when $x_{m}$ obtained from (1a in step 3) exceeds the tolerance value of them component, and fault component is the component $m$

\section{SIMULATED RESULTS}

It is in general to employ a three tone test for the analog circuits. For this purpose, it is suggested to use a low frequency tone, a tone around corner frequency, and a high tone frequency close to the stop band frequency as a good combination for the three tone test. As per design specifications, select $22 \mathrm{kHz}, 51 \mathrm{kHz}$, and $398 \mathrm{kHz}$ as the stimulus tones. All the circuit simulations are done using Mentor Graphics and tanner $0.25 \mu \mathrm{m}$ technology EDA tools.

Fig.7 represents the schematic view of fifth order low pass filter. Fig. 8 shows the simulation results of the CUT when the parametric fault of $\bar{C}_{G p f}$ is injected. This parametric fault makes the pass band gain and the stop band attenuation of the CUT out of the design specification. Even 
though the CUT fails in the test, the estimated TF still accurately Yet the estimated TFs successfully depicts the faulty frequency responses of the CUT.Fig.13 shows the frequency response of fifth order low pass filter ,from that the pass band frequency of $8 \mathrm{MHz}$,pass band ripple is $<1 \mathrm{~dB}, 80 \mathrm{MHz}$ clock frequency at the supply voltage of $1.8 \mathrm{~V}$ and stop band attenuation is higher than $40 \mathrm{~dB}$,

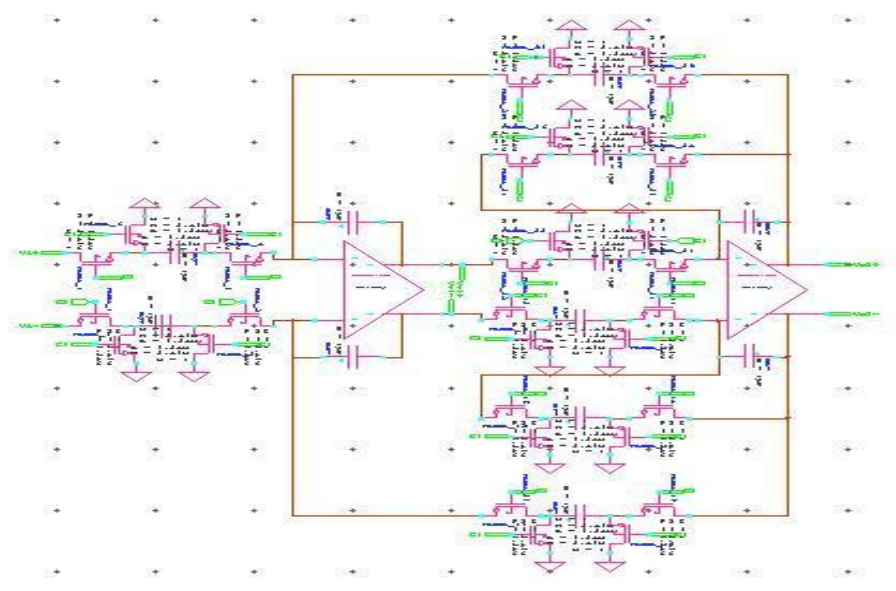

Fig 6: Biquad filter using second order low pass filter

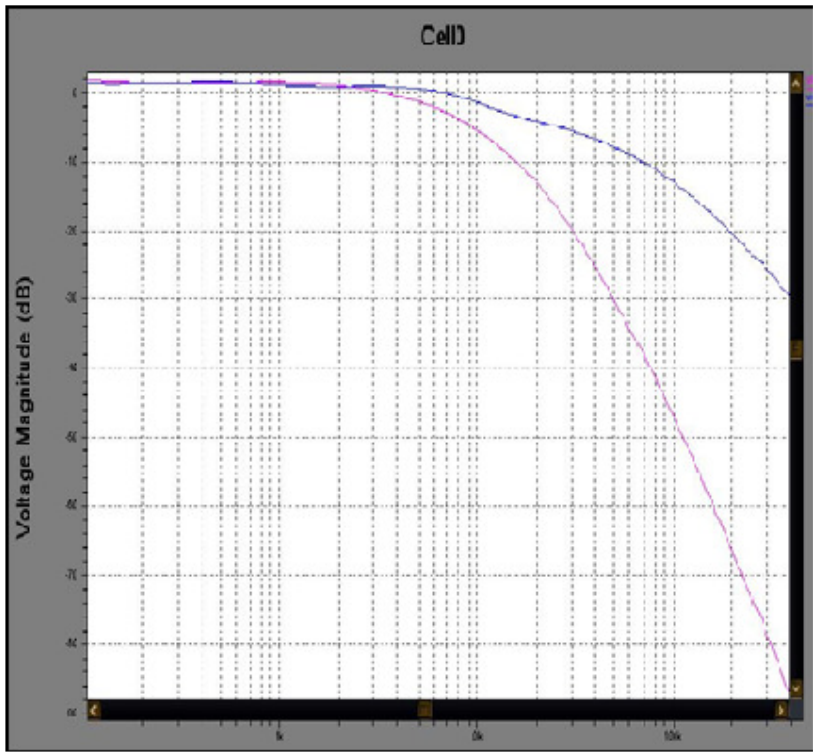

Fig 7:Frequency response of biquad filter in second order filter 
International Journal on Cybernetics \& Informatics (IJCI) Vol. 5, No. 4, August 2016

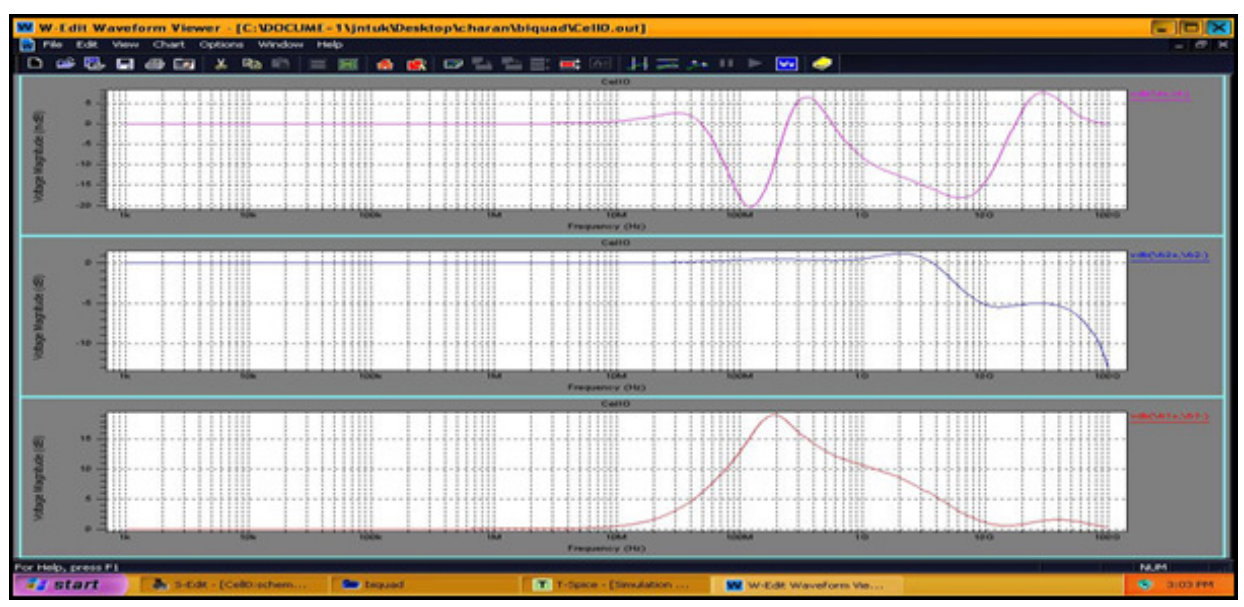

Fig8: Biquad filter output of without injecting any fault

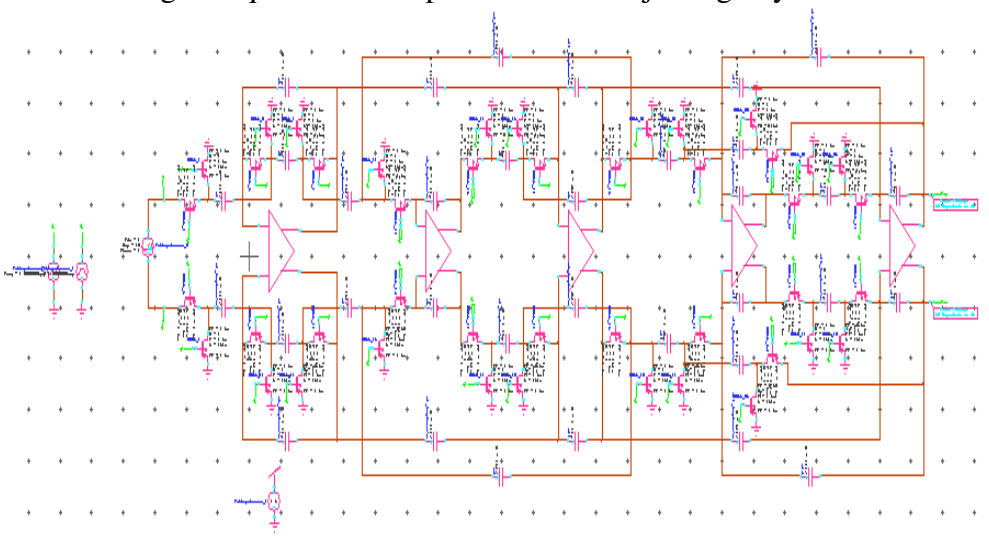

Fig 9. Schematic view of fifth order SC low pass filter

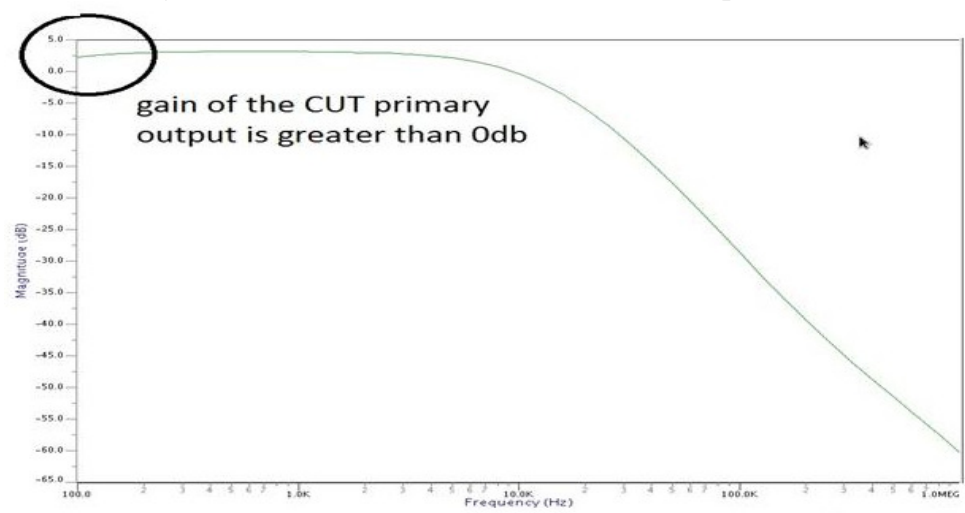

Fig 10. Experimental result after injecting parametric fault $C_{G p f}$. 
International Journal on Cybernetics \& Informatics (IJCI) Vol. 5, No. 4, August 2016

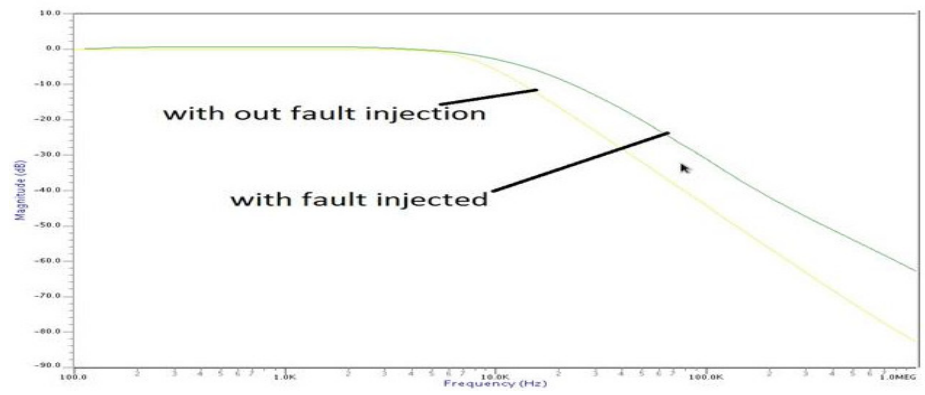

Fig 11. Experimental result after injecting parametric fault Ap

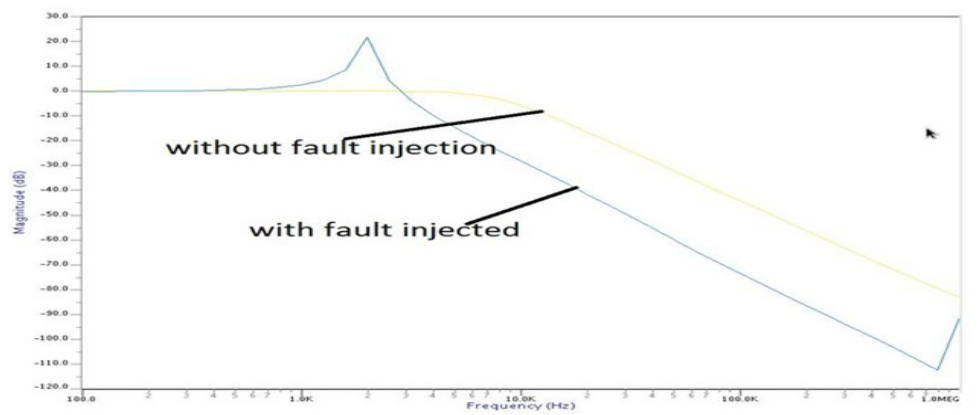

Fig 12. Experimental result after injecting parametric fault Ep.

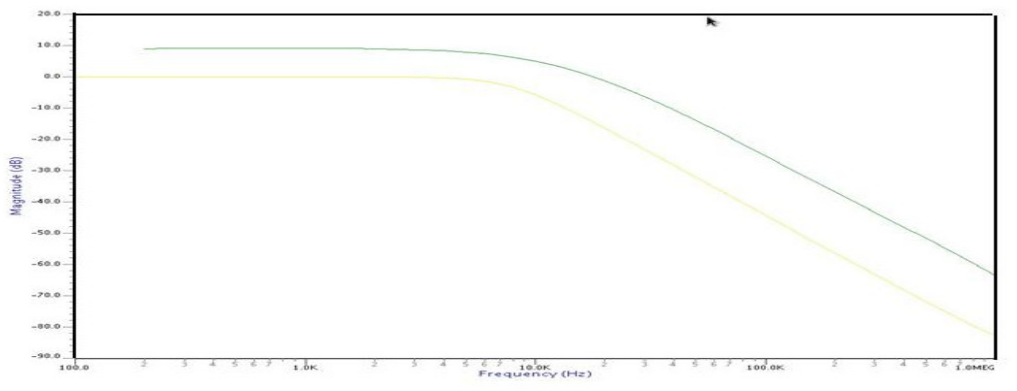

Fig 13. Experimental result when switch SHs is short.

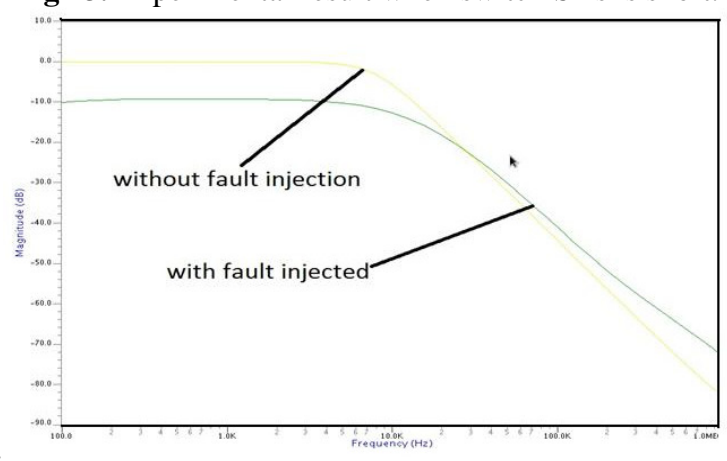


Fig 14. Experimental result when switch SGo is open

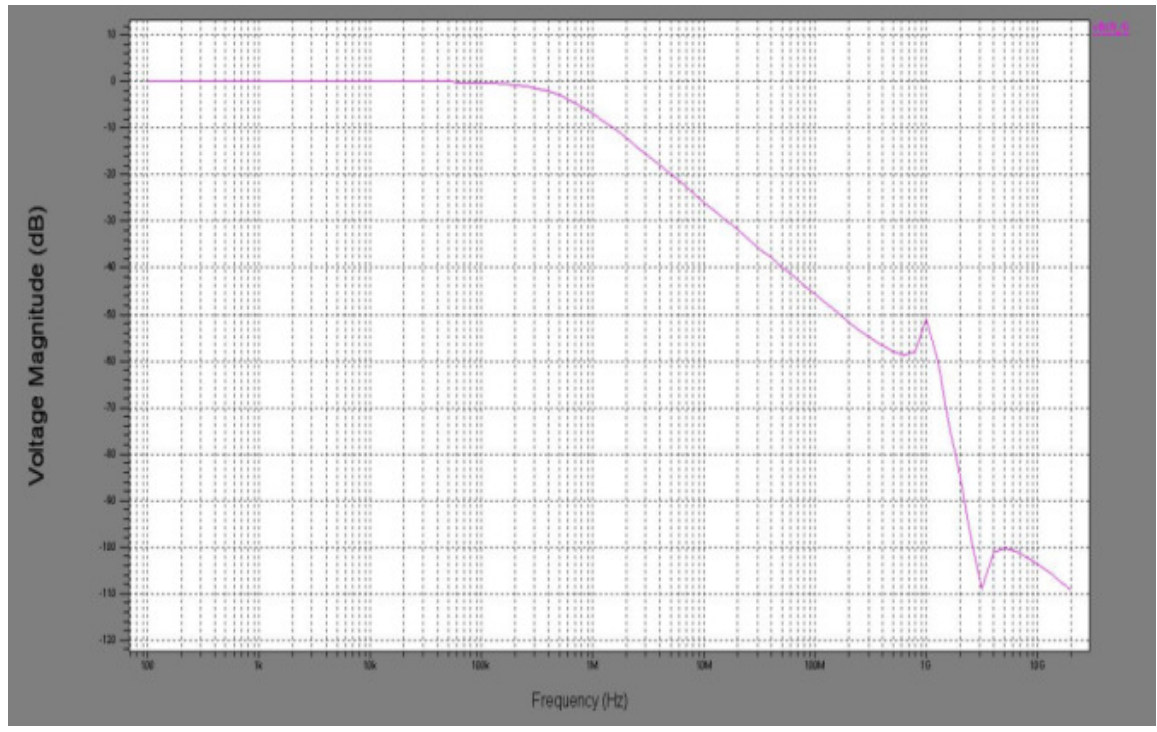

Fig 15 Frequency response of the SC low pass filter.

\section{VECTORISATION METHOD RESULTS}

\begin{tabular}{|l|c|c|c|c|c|}
\hline \multicolumn{1}{|c|}{ faults } & $\begin{array}{c}\text { vma } \\
\mathrm{x} \\
\text { vo1 }\end{array}$ & Vo2 & $\begin{array}{c}\text { vmin } \\
\text { Vo1 }\end{array}$ & Vo2 & $\begin{array}{c}\text { Coefficient } \\
\text { s } \\
\mathrm{K}, \mathrm{b}\end{array}$ \\
\hline $\begin{array}{l}\text { Capacitor } \\
\text { enable }\end{array}$ & 4.6 & 7.04 & -80 & -65 & $\begin{array}{c}-523.906 \\
525.0129\end{array}$ \\
\hline $\begin{array}{l}\text { Capacitor } \\
\text { ground }\end{array}$ & 3.88 & 8.4 & -70.2 & -75 & $\begin{array}{c}-711.4063 \\
712.5129\end{array}$ \\
\hline $\begin{array}{c}\text { Switches } \\
\text { short(NMOS } \\
\text { ) }\end{array}$ & -104 & 3.88 & -4 & --70 & $\begin{array}{c}-804.0562 \\
805.1629\end{array}$ \\
\hline $\begin{array}{c}\text { Switches } \\
\text { open(NMOS } \\
\text { ) }\end{array}$ & -111 & -0.8 & -71.1 & -1.6 & $\begin{array}{c}-219.9063 \\
218.7996\end{array}$ \\
\hline
\end{tabular}

Fig.4 Faults table in eigenvectors

This result show the coefficients of eigen values of biquad filter circuit it can determine the in which component the can seen in this table

\section{CONCLUSiON}

In this paper the SLB simple shortcoming model for straight SC circuits is been checked and a SC low pass biquad channel is taken as a case to show the adequacy of the SLB issue model. We lead with different stimulus tones and check if the test reactions fit in those anticipated by the recovered TF. Trial results check that the settled TF layout supposition holds for every one of the 
issues that we infused. Extending the shortcoming model to incorporate the planning related deficiencies would be a fascinating subject for the future exploration work.

\section{REFERENCES}

[1] H.C. Hong, "A Static Linear Behavior Analog Fault Model for Switched-Capacitor Circuits," IEEE Trans. Computer-Aided Design of Integrated Circuits and Systems, vol. 31, no. 4, pp. 597-609, 2012.

[2] Long-Yi Lin and Hao-Chiao Hong," Design of a Fault-Injectable Fleischer-Laker Switched-Capacitor Biquad for verifying the Static Linear Behavior Fault Model”, 22nd Asian Test Symposium, 2013

[3] M. Fino, J. Franca, and A. Steiger-Garcao, "Automatic symbolic analysis of switched-capacitor filtering networks using signal flow graphs,” IEEE Trans. Comput.-Aided Des. Integr. Circuits Syst., vol. 14, no. 7, pp. 858-867, Jul. 1995.

[4] S. F. Hung, L. Y. Lin, and H.-C. Hong, "A study on the Design of a Testable Fleisher-Laker Switched-Capacitor Biquad," in Proc. Int.Mixed-Signals, Sensors,and System Testing Workshop (IMS3TW), 2012,pp. 119-122.

[5] Priyanka Kakoty "Design of a high frequency low voltage CMOS operational amplifier,"International Journal of VLSI design \& Communication Systems (VLSICS) Vol.2, No.1, March 2011. 